\title{
DIVISION X WORKING GROUP on RADIO FREQUENCY INTERFERENCE MITIGATION
}

\author{
CHAIR \\ VICE-CHAIR \\ PAST CHAIR \\ MEMBERS
}

\author{
Tasso Tzioumis (AUS) \\ Willem Baan (NL), Darrel Emerson USA), \\ Masatoshi Ohishi) Japan), Tomas Gergely) USA), \\ Rick Fisher (USA), John Ponsoby (UK), \\ JAlbert-Jan Boonstra (NL), Ron Ekers (AUS), \\ Wim van Driel (France), Haiyan Zhang (China)
}

\section{PROCEEDINGS BUSINESS SESSION, 6 August 2009}

\section{Introduction}

The IAU Working Group on Radio Frequency Interference (RFI) Mitigation was setup in the 2000 IAU GA in Manchester and its mandate was renewed at subsequent IAU GAs in 2003 and 2006. It was noted that that there are important issues related to RFI mitigation that extend beyond the regulatory function of IUCAF, and hence a more extended working group, which may include IUCAF members, was established.

\section{Terms of Reference}

The IAU Working Group on RFI mitigation was tasked to seek:

1. Technological solutions: inter alia, interference rejection schemes, novel types of modulation manifesting inherently low out-of-band emissions, state-of-the-art RF filter technology and how it may be advanced, antenna null steering, interference recognition, and data editing.

2. Regulatory innovations: new ways of sharing the radio spectrum.

3. Radio-quiet zones: designating remote areas on the Earth's surface where satellite and other broadcasts (emissions) will be restricted in frequency and in time, and where future radio observatories may be located.

4. Institutional innovations: supranational body to examine and test all space vehicles for out-of-band and spurious emissions prior to launch and with power to arrest launch in event of unsatisfactory performance; also, ways the ITU might be constrained by the 1967 Outer Space Treaty and the 1972 Liability Convention and by its obligation to submit itself to higher authority within the UN family of organizations in the event of its interests conflicting with those of other bodies of equal standing in the UN family of organizations.

The terms of reference were revised in 2006 and no further revision was suggested at this meeting.

\section{Report on recent progress with technological solutions}

\subsection{Workshops}

The last major workshop on RFI mitigation was at the 2004 Penticton SKA meeting (RFI2004), as reported in the previous IAU GA. It was cosponsored by IUCAF and selected contributions were published in Radio Science, 40 (2005). In 2008 a session on "Radio Frequency Interference, Problems and Solutions" was held at the URSI GA (Chicago, August 2008). 
An RFI mitigation workshop was planned for 2007 but it did not eventuate. The next workshop (RFI2010) will be held in Gronigen, the Netherlands, on 29-31 March 2010. A first announcement has been circulated but details may change.

\subsection{Reports on RFI Mitigation techniques}

This working group has traditional commissioned reports on RFI mitigation techniques from researchers in this field. These reports have been presented and distributed at each IAU GA. Previous reports by Geoff Bower (USA) in 2003 and Steve Ellingson (USA) in 2006 are available via the Division $\mathrm{X}$ website.

A new report by Michael Kesteven (Australia) was commissioned for this IAU GA and it is available in hardcopy and on the IAU website. The subjects covered in this report are given in the following outline:

The Current Status of RFI Mitigation in Radioastronomy

- RFI categories - Satellites; Aircraft; Ground-based; Observatory-based

- Proactive Mitigation strategies

- Regulation

- Radio Quiet Zones

- The Observatory environment

- Reactive Mitigation strategies

- Blanking in time; in frequency; flagging

- Null steering

- Adaptive filters

- Mitigation in the array imaging stage

- Subspace filtering

- Generalised spatial filtering

- RFI Mitigation Metrics

- Discussion on Uptake and SKA

- References

The report includes an extensive list of recent references in this field.

\section{Regulatory innovations}

Regulatory measures are the domain of the International Telecommunications Union (ITU) and a Question on RFI mitigation exists in the ITU-R Study Group 7. Work continues towards the production of a New Report on RFI Mitigation in the ITU-R but political issues and sensitivities need to be addressed.

To facilitate education in the spectrum regulatory environment, IUCAF and other astronomy institutions have been cosponsoring "Summer Schools on Spectrum Management". The previous such summer school was co-sponsored by RadioNet and was conducted in Bologna in 2005. A summer school was in planning for 2008-9 but fell victim to the world financial crisis. The next summer school on spectrum management is planned for early 2010 in Japan.

\section{Radio Quiet Zones (RQZ)}

Radio Quiet Zones have been used to protect current radio astronomy facilities in many countries. RQZs were also recommended by the OECD Global Science Forum "Task Force on Radio Astronomy and the Radio Spectrum" in 2003. Work in this subject continues in two areas:

(a) A Question on RQZ has been raised at the ITU-R and studies are underway towards in ITU-R Report. This issue may become a future item in a World Radio Conference (WRC) but the politics need to be delicately handled.

(b) The Square Kilometre Array (SKA), the next generation radio astronomy facility, requires a RQZ. A SKA task group has produced a report on the requirements of a RQZ for the SKA. Both candidate SKA sites in Australia and South Africa have established RQZs to protect current pathfinder instruments (ASKAP; meerKAT) and the SKA in the future. Technical work in this area will continue by the SKA project. 


\section{Discussion on mitigation uptake and the SKA}

The report by M. Kesteven raised some interesting questions that were discussed by the group:

Low level of uptake of RFI mitigation: Despite significant technological developments in RFI mitigation few observatories have implemented on-line, routine RFI mitigation strategies. It appears that traditional simple techniques such as blanking, discarding badly affected data blocks, or rescheduling are the fallback mitigation options.

There appear to be many reasons for this which include the high cost of mitigation and the application of "niche" solutions since there is no universal mitigation technique. It was felt that it is imperative to continue research as RFI is getting worse and mitigation may become critical in the future.

RFI mitigation and the SKA: It is likely that, due to the RQZ on SKA sites and the high computational cost of mitigation techniques, the early SKA may not include RFI mitigation. However, it was strongly felt that the deteriorating RFI environment would necessitate mitigation in the future and research in RFI mitigation for the SKA is essential.

\section{Review and discussion on the future of this working group}

The meeting reviewed the work of this Working Group, which is primarily reporting on research and other developments in RFI mitigation achieved by other groups. The WG also encourages and cosponsors workshops and reports, even though the WG has few direct resources to allocate.

It was expressed strongly in the meeting that, given that RFI is getting worse and mitigation is becoming more important, the IAU should continue with the working group.

The present chair (Tasso Tzioumis) stood down after 9 years and Willem Baan was appointed the new chair of the working group.

\section{Concluding remarks and suggested actions}

There was strong support in continuing this working group and it was felt that RFI mitigation will become progressively more important and even critical. The meeting also suggested the following actions for the WG:

- Encourage submission of a suitably modified version of the technical report to the ITU-R for inclusion in its report.

- Help establish a database of references and papers in this field. The references in the technical reports should provide a good start.

- Investigate whether the WG should help organise an IAU Symposium on this subject, perhaps at the next GA.

- Investigate inclusion in the work of this WG of protection of optical/IR dark skies, in consultation and collaboration with IAU Commission 50. 